\title{
Article
}

\section{Global well-posedness and analyticity for generalized porous medium equation in critical Fourier-Besov-Morrey spaces}

\author{
Mohamed Toumlilin ${ }^{1, *}$ \\ 1 FST FES, Laboratory AAFA, Department of Mathematics, University Sidi Mohamed Ben Abdellah, Fes, Morocco. \\ * Correspondence: mohamed.toumlilin@usmba.ac.ma
}

Received: 23 July 2019; Accepted: 29 September 2019; Published: 19 October 2019.

\begin{abstract}
In this paper, we study the generalized porous medium equations with Laplacian and abstract pressure term. By using the Fourier localization argument and the Littlewood-Paley theory, we get global well-posedness results of this equation for small initial data $u_{0}$ belonging to the critical Fourier-Besov-Morrey spaces. In addition, we also give the Gevrey class regularity of the solution.
\end{abstract}

Keywords: Porous medium equation, well-posedness, analyticity, Fourier-Besov-Morrey space.

MSC: 35K55, 74G25,76S05.

\section{Introduction}

W

e investigate the generalized porous medium equation in the whole space $\mathbb{R}^{3}$,

$$
\left\{\begin{array}{l}
u_{t}+\mu \Lambda^{\alpha} u=\nabla \cdot(u \nabla P u) ; \quad(t, x) \in \mathbb{R}^{+} \times \mathbb{R}^{3}, \\
u(0, x)=u_{0} \quad x \in \mathbb{R}^{3}
\end{array}\right.
$$

where $u=u(t, x)$ is a real-valued function, which denotes a density or concentration. The dissipative coefficient $\mu>0$ corresponds to the viscous case, while $\mu=0$ corresponds to the inviscid case. The fractional Laplacian operator $\Lambda^{\alpha}$ is defined by Fourier transform as $\widehat{\Lambda^{\alpha}} u=|\xi|^{\alpha} \hat{u}$, and $P$ is an abstract operator.

The equation (1) was introduced in the first by Zhou et al. [1]. In fact, Equation (1) is obtained by adding the fractional dissipative term $\mu \Lambda^{\alpha} u$ to the continuity equation (PME) $u_{t}+\nabla \cdot(u V)=0$ given by Caffarelli and Vázquez [2], where the velocity $V$ derives from a potential, $V=-\nabla p$ and the velocity potential or pressure $p$ is related to $u$ by an abstract operator $p=P u[3]$.

For $\mu=0$ and $P u=(-\Delta)^{-s} u=\Lambda^{-2 s} u, 0<s<1$; X. Zhou et al. [4] were interested in finding the strong solutions of the equation (1) which becomes the fractional porous medium equation in the Besov spaces $B_{p, \infty}^{\alpha}$ and they obtained the local solution for any initial data in $B_{1, \infty}^{\alpha}$. Moreover, in the critical case $s=1$, the Equation (1) leads to a mean field equation [4,5]. Let's take this opportunity to briefly quote some works on the well-posedness and regularity on those equations such as $[4,6]$ and the references therein.

On the other hand, an another similar model occurs in the aggregation equation, and plays a fundamental role in applied sciences such as physics, biology, chemistry, population dynamics. It describes a collective motion and aggregation phenomena in biology and in mechanics of continuous media $[7,8]$. In the aggregation equation, the abstract form pressure term $P u$ can also be represented by convolution with a kernel $K$ as $P u=$ $K * u$. The typical kernels are the Newton potential $|x|^{\gamma}[9,10]$, and the exponent potential $-e^{-|x|}[11,12]$. For more results on this equation, we refer to $[13,14]$ and the references therein.

Recently, Zhou et al. [1] obtained the local well-posedness in Besov spaces for large initial data, and proved that the solution becomes global if the initial data is small, also, they studied a blowup criterion for the solution. 
In addition, we can represent the Equation (1) with the same initial data by

$$
\begin{gathered}
u_{t}+\mu \Lambda^{\alpha} u+v \cdot \nabla u=-u(\nabla \cdot v) \\
v=-\nabla P u
\end{gathered}
$$

As a consequence, this equation must be compared to the geostrophic model. So, the convective velocity is not absolutely divergence-free for the generalized porous medium equation. Additionally, if we assume that $v$ is divergence-free vector function $(\nabla \cdot v=0)$, the form (2) can contain the quasi-geostrophic (Q-G) equation $[15,16]$.

Inspired by the works [1,17]; the aim of this paper is to prove the well-posedness results of Equation (1) and to give the Gevrey class regularity of the solution in homogeneous Fourier Besov-Morrey spaces under the condition that the abstract operator $P$ is commutative with the operator $e^{-\mu \sqrt{t}|D|^{\frac{\alpha}{2}}}$ and

$$
\left\|\varphi_{j} \widehat{\nabla P u}\right\|_{\mathbf{M}_{p}^{\lambda}} \leq C 2^{j \sigma}\left\|\varphi_{j} \widehat{u}\right\|_{\mathbf{M}_{p}^{\lambda}}
$$

Clearly, for the fractional porous medium equation, i.e. $P u=\Lambda^{-2 s} u$, we get $\sigma=1-2 s$. If $P u=K * u$ in the aggregation equation, $W u$ and Zhang [18] proved a similar result under the condition $\nabla K \in W^{1,1}$, $\alpha \in(0,1)$. Corresponding to their case we give a same result for $\sigma=0$ when $\nabla K \in L^{1}$, and also a similar result for $\sigma=1$ when $K \in L^{1}$.

Throughout this paper, we use $\mathcal{F} \dot{\mathcal{N}}_{p, \lambda, q}^{s}$ to denote the homogenous Fourier Besov-Morrey spaces, $C$ will denote constants which can be different at different places, $\mathrm{U} \lesssim \mathrm{V}$ means that there exists a constant $C>0$ such that $\mathrm{U} \leq \mathrm{CV}$, and $p^{\prime}$ is the conjugate of $p$ satisfying $\frac{1}{p}+\frac{1}{p^{\prime}}=1$ for $1 \leq p \leq \infty$.

\section{Preliminaries and main results}

We start with a dyadic decomposition of $\mathbb{R}^{n}$. Suppose $\chi \in C_{0}^{\infty}\left(\mathbb{R}^{n}\right), \varphi \in C_{0}^{\infty}\left(\mathbb{R}^{n} \backslash\{0\}\right)$ satisfying

$$
\begin{gathered}
\operatorname{supp} \chi \subset\left\{\xi \in \mathbb{R}^{n}:|\xi| \leq \frac{4}{3}\right\}, \\
\operatorname{supp} \varphi \subset\left\{\xi \in \mathbb{R}^{n}: \frac{3}{4} \leq|\xi| \leq \frac{8}{3}\right\}, \\
\chi(\xi)+\sum_{j \geq 0} \varphi\left(2^{-j} \xi\right)=1, \quad \xi \in \mathbb{R}^{n}, \\
\sum_{j \in \mathbb{Z}} \varphi\left(2^{-j} \xi\right)=1, \quad \xi \in \mathbb{R}^{n} \backslash\{0\},
\end{gathered}
$$

and denote $\varphi_{j}(\xi)=\varphi\left(2^{-j} \xi\right)$ and $\mathcal{P}$ the set of all polynomials.

First, we recall the definition of Morrey spaces which are a complement of $L^{p}$ spaces.

Definition 1 ([19]). For $1 \leq p<\infty, 0 \leq \lambda<n$, the Morrey spaces $\mathrm{M}_{p}^{\lambda}=\mathrm{M}_{p}^{\lambda}\left(\mathbb{R}^{n}\right)$ is defined as the set of functions $f \in L_{l o c}^{p}\left(\mathbb{R}^{n}\right)$ such that

$$
\|f\|_{\mathbf{M}_{p}^{\lambda}}=\sup _{x_{0} \in \mathbb{R}^{n}} \sup _{r>0} r^{-\frac{\lambda}{p}}\|f\|_{L^{p}\left(B\left(x_{0}, r\right)\right)}<\infty,
$$

where $B\left(x_{0}, r\right)$ denotes the ball in $\mathbb{R}^{n}$ with center $x_{0}$ and radius $r$.

It is easy to see that the injection $\mathrm{M}_{p_{1}}^{\lambda} \hookrightarrow \mathrm{M}_{p_{2}}^{\mu}$ provided $\frac{n-\mu}{p_{2}} \geq \frac{n-\lambda}{p_{1}}$ and $p_{2} \leq p_{1}$, and $\mathrm{M}_{p}^{0}=L^{p}$.

If $1 \leq p_{1}, p_{2}, p_{3}<\infty$ and $0 \leq \lambda_{1}, \lambda_{2}, \lambda_{3}<n$ with $\frac{1}{p_{3}}=\frac{1}{p_{1}}+\frac{1}{p_{2}}$ and $\frac{\lambda_{3}}{p_{3}}=\frac{\lambda_{1}}{p_{1}}+\frac{\lambda_{2}}{p_{2}}$, then we have the Hölder type inequality

$$
\|f g\|_{\mathrm{M}_{p_{3}}^{\lambda_{3}}} \leq\|f\|_{\mathrm{M}_{p_{1}}^{\lambda_{1}}}\|g\|_{\mathrm{M}_{p_{2}}^{\lambda_{2}}} .
$$

Also, for $1 \leq p<\infty$ and $0 \leq \lambda<n$,

$$
\|\varphi * g\|_{\mathrm{M}_{p}^{\lambda}} \leq\|\varphi\|_{L^{1}}\|g\|_{\mathrm{M}_{p}^{\lambda}}
$$


for all $\varphi \in L^{1}$ and $g \in \mathbf{M}_{p}^{\lambda}$.

Definition 2. (homogeneous Fourier-Besov-Morrey spaces ) Let $s \in \mathbb{R}, 0 \leq \lambda<n, 1 \leq p<+\infty$ and $1 \leq q \leq+\infty$. The space $\mathcal{F} \dot{\mathcal{N}}_{p, \lambda, q}^{\mathcal{S}}\left(\mathbb{R}^{n}\right)$ denotes the set of all $u \in \mathcal{S}^{\prime}\left(\mathbb{R}^{n}\right) / \mathcal{P}$ such that

$$
\|u\|_{\mathcal{F N}_{p, \lambda, q}^{s}\left(\mathbb{R}^{n}\right)}=\left\{\sum_{j \in \mathbb{Z}} 2^{j q s}\left\|\varphi_{j} \widehat{u}\right\|_{\mathrm{M}_{p}^{\lambda}}^{q}\right\}^{1 / q}<+\infty,
$$

with suitable modification made when $q=\infty$.

Note that the space $\mathcal{F} \dot{\mathcal{N}}_{p, \lambda, q}^{s}\left(\mathbb{R}^{n}\right)$ equipped with the norm (6) is a Banach space. Since $\mathrm{M}_{p}^{0}=L^{p}$, we have $\mathcal{F} \dot{\mathcal{N}}_{p, 0, q}^{s}=F \dot{B}_{p, q}^{s}, \mathcal{F N}_{1,0, q}^{s}=F \dot{B}_{1, q}^{s}=\dot{\mathcal{B}}_{q}^{s}$ and $\mathcal{F} \dot{\mathcal{N}}_{1,0,1}^{-1}=\chi^{-1}$ where $\dot{\mathcal{B}}_{q}^{s}$ is the Fourier-Herz space and $\chi^{-1}$ is the Lei-Lin space [20].

Now, we recall the definition of the mixed space-time spaces.

Definition 3. Let $s \in \mathbb{R}, 1 \leq p<\infty, 1 \leq q, \rho \leq \infty, 0 \leq \lambda<n$, and $I=[0, T), T \in(0, \infty]$. The space-time norm is defined on $u(t, x)$ by

$$
\|u(t, x)\|_{\mathcal{L}^{\rho}\left(I ; \mathcal{F} \dot{\mathcal{N}}_{p, \lambda, q}^{s}\right)}=\left\{\sum_{j \in \mathbb{Z}} 2^{j q s}\left\|\varphi_{j} \widehat{u}\right\|_{L^{\rho}\left(I, \mathrm{M}_{p}^{\lambda}\right)}^{q}\right\}^{1 / q}
$$

and denote by $\mathcal{L}^{\rho}\left(I ; \dot{\mathcal{N}}_{p, \lambda, q}^{s}\right)$ the set of distributions in $S^{\prime}\left(\mathbb{R} \times \mathbb{R}^{n}\right) / \mathcal{P}$ with finite $\|\cdot\|_{\mathcal{L}^{\rho}\left(I ; \mathcal{F} \dot{\mathcal{N}}_{p, \lambda, q}^{s}\right)}$ norm.

According to Minkowski inequality, we have

$$
\begin{array}{ll}
L^{\rho}\left(I ; \mathcal{F} \dot{\mathcal{N}}_{p, \lambda, q}^{s}\right) \hookrightarrow \mathcal{L}^{\rho}\left(I ; \mathcal{F} \dot{\mathcal{N}}_{p, \lambda, q}^{s}\right), & \text { if } \rho \leq q, \\
\mathcal{L}^{\rho}\left(I ; \mathcal{F} \dot{\mathcal{N}}_{p, \lambda, q}^{s}\right) \hookrightarrow L^{\rho}\left(I ; \mathcal{F} \dot{\mathcal{N}}_{p, \lambda, q}^{s}\right), & \text { if } \rho \geq q,
\end{array}
$$

where $\|u(t, x)\|_{L^{\rho}\left(I ; \mathcal{F} \dot{\mathcal{N}}_{p, \lambda, q}^{s}\right)}:=\left(\int_{I}\|u(\tau, \cdot)\|_{\mathcal{F}_{\mathcal{N}_{p, \lambda, q}^{s}}^{\rho}}^{\rho} d \tau\right)^{1 / \rho}$.

Our first main result is the following theorem.

Theorem 4. Assume that the abstract operator $P$ satisfies the condition (3). If $0 \leq \lambda<3,1 \leq q \leq \infty, 1 \leq p<\infty$ and $\max \{1+\sigma, 0\}<\alpha<2+\frac{3}{p^{\prime}}+\frac{\lambda}{p}+\sigma$ then there exists a constant $C_{0}$ such that for any $u_{0} \in \mathcal{F}_{\mathcal{N}^{1}, \lambda, q}^{1-\alpha+\frac{3}{p^{\prime}}+\frac{\lambda}{p}+\sigma}$ satisfies $\left\|u_{0}\right\| \underset{\mathcal{F N}_{p, \lambda, q}^{1-\alpha+\frac{3}{p^{\prime}}+\frac{\lambda}{p}+\sigma}}{ }<C_{0} \mu$, the equation(1) admits a unique global solution $u$,

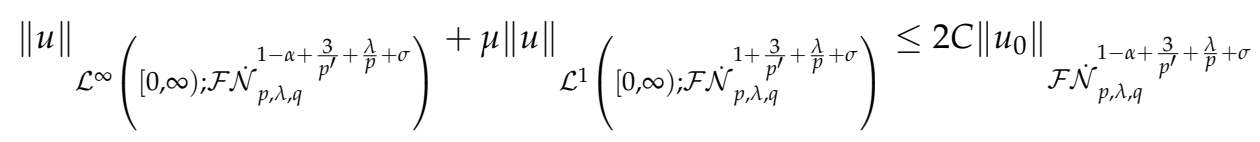

where $C$ is a positive constant.

Now, we give some remarks about this result.

Remark 1. The result stated in Theorem 4 is based on the works [3]. In particular, this result remains true if we replace the Fourier-Besov-Morrey space $\mathcal{F} \dot{\mathcal{N}}_{p, \lambda, q}^{s}$ by other functional spaces such as Fourier-Herz space $\dot{\mathcal{B}}_{q}^{s}$, Fourier-Besov space $\mathrm{FB}_{p, q}^{s}$ and Lei-Lin space $\chi^{-1}$.

The analyticity of the solution is also an important subject developed by several researchers, particularly with regard to the Navier-Stokes equations, see [17] and its references. In this paper, we will prove the Gevrey class regularity for (1) in the Fourier-Besov-Morrey space. Inspired by this, we have obtained the following specific results. 
Theorem 5. Let $0 \leq \lambda<3,1 \leq q \leq \infty, 1 \leq p<\infty$ and $\max \{1+\sigma, 0\}<\alpha<\min \left\{2,2+\frac{3}{p^{\prime}}+\frac{\lambda}{p}+\sigma\right\}$. There exists a constant $C_{0}$ such that, if $u_{0} \in \mathcal{F} \dot{\mathcal{N}}_{p, \lambda, q}^{1-\alpha+\frac{3}{p^{\prime}}+\frac{\lambda}{p}+\sigma}$ satisfies $\left\|u_{0}\right\|_{\mathcal{F N}_{p, \lambda, q}^{1-\alpha+\frac{3}{p^{2}}}+\frac{\lambda}{p}+\sigma}<C_{0} \mu$, then the Cauchy problem

(1) admits a unique analytic solution $u$, in the sense that

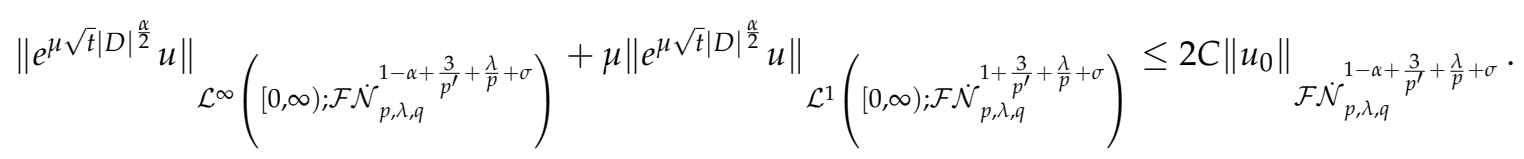

We finish this section with a Bernstein type lemma in Fourier variables in Morrey spaces.

Lemma 6 ([21]). Let $1 \leq q \leq p<\infty, 0 \leq \lambda_{1}, \lambda_{2}<n, \frac{n-\lambda_{1}}{p} \leq \frac{n-\lambda_{2}}{q}$, and let $\gamma$ be a multiindex. If supp $(\widehat{f}) \subset$ $\left\{|\xi| \leq A 2^{j}\right\}$ then there is a constant $C>0$ independent of $f$ and $j$ such that

$$
\left\|(i \xi)^{\gamma} \widehat{f}\right\|_{\mathrm{M}_{q}^{\lambda_{2}}} \leq C 2^{j|\gamma|+j\left(\frac{n-\lambda_{2}}{q}-\frac{n-\lambda_{1}}{p}\right)}\|\widehat{f}\|_{\mathrm{M}_{p}^{\lambda_{1}}} .
$$

\section{The well-posedness}

First, we consider the linear nonhomogeneous dissipative equation

$$
\left\{\begin{array}{l}
u_{t}+\mu \Lambda^{\alpha} u=f(t, x) \quad(t, x) \in \mathbb{R}^{+} \times \mathbb{R}^{3} \\
u(0, x)=u_{0}(x) \quad x \in \mathbb{R}^{3}
\end{array}\right.
$$

for which we recall the following result.

Lemma 7 ([22]). Let $I=[0, T), 0<T \leq \infty, s \in \mathbb{R}, 0 \leq \lambda<3,1 \leq p<\infty$, and $1 \leq q, \rho \leq \infty$. Assume that $u_{0} \in \mathcal{F} \dot{\mathcal{N}}_{p, \lambda, q}^{s}$ and $f \in \mathcal{L}^{\rho}\left(I ; \mathcal{F}_{\mathcal{N}_{p, \lambda, q}^{s-\alpha+\frac{\alpha}{\rho}}}\right)$. Then the Cauchy problem (8) has a unique solution $u(t, x)$ such that for all $\rho_{1} \in[\rho,+\infty]$

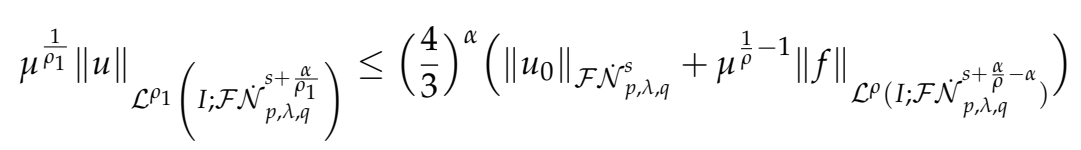

and

$$
\|u\|_{\mathcal{L}^{\infty}\left(I ; \mathcal{F} \dot{\mathcal{N}}_{p, \lambda, q}^{s}\right)}+\mu\|u\|_{\mathcal{L}^{1}\left(I ; \mathcal{F} \dot{\mathcal{N}}_{p, \lambda, q}^{s+\alpha}\right)} \leq\left(1+\left(\frac{4}{3}\right)^{\alpha}\right)\left(\left\|u_{0}\right\|_{\mathcal{F N}_{p, \lambda, q}^{s}}+\|f\|_{\mathcal{L}^{1}\left(I ; \mathcal{F} \dot{\mathcal{N}}_{p, \lambda, q}^{s}\right)}\right)
$$

If in addition $q$ is finite, then $u$ belongs to $\mathcal{C}\left(I ; \mathcal{F}_{\mathcal{N}_{p, \lambda, q}^{s}}^{s}\right)$.

Proposition 8. Let $1 \leq p<\infty, 1 \leq \rho, q \leq \infty, 1+\sigma<\alpha<\frac{2+\frac{3}{p^{\prime}}+\frac{\lambda}{p}+\sigma}{2-\frac{1}{\rho}}, 0 \leq \lambda<3, I=[0, T), T \in(0, \infty]$, and set

$$
X=\mathcal{L}^{\infty}\left(I ; \mathcal{F} \dot{\mathcal{N}}_{p, \lambda, q}^{1-\alpha+\frac{3}{p^{\prime}}+\frac{\lambda}{p}+\sigma}\right) \cap \mathcal{L}^{\rho}\left(I ; \mathcal{F}_{p, \lambda, q}^{1-\alpha+\frac{3}{p^{\prime}}+\frac{\lambda}{p}+\frac{\alpha}{\rho}+\sigma}\right)
$$

with the norm

$$
\|u\|_{X}=\|u\|_{\mathcal{L}^{\infty}\left(I ; \mathcal{F} \dot{\mathcal{N}}_{p, \lambda, q}^{1-\alpha+\frac{3}{p^{\prime}}+\frac{\lambda}{p}+\sigma}\right)}+\mu\|u\| \mathcal{L}^{\rho}\left(I ; \mathcal{F} \dot{\mathcal{N}}_{p, \lambda, q}^{1-\alpha+\frac{3}{p^{\prime}}+\frac{\lambda}{p}+\frac{\alpha}{\rho}+\sigma}\right) .
$$

There exists a constant $C=C(p, q)>0$ depending on $p, q$ such that

$$
\left\|u \partial_{i} P v\right\|_{\mathcal{L}^{\rho}\left(I ; \mathcal{F} \dot{\mathcal{N}}_{p, \lambda, q}^{-2(\alpha-1)+\frac{3}{p^{\prime}}+\frac{\alpha}{\rho}+\frac{\lambda}{p}+\sigma}\right)} \leq C \mu^{-1}\|u\|_{X}\|v\|_{X} .
$$


Proof. Let us introduce some notations about the standard localization operators. We set

$$
u_{j}=\dot{\Delta}_{j} u=\left(\mathscr{F}^{-1} \varphi_{j}\right) * u, \quad \dot{S}_{j} u=\sum_{k \leq j-1} \dot{\Delta}_{k} u, \quad \widetilde{\dot{\Delta}}_{j} u=\sum_{|k-j| \leq 1} \dot{\Delta}_{k} u, \quad \forall j \in \mathbb{Z} .
$$

Using the decomposition of Bony's paraproducts for the fixed $j$, we have

$$
\begin{aligned}
\dot{\Delta}_{j}\left(u \partial_{i} P v\right) & =\sum_{|k-j| \leq 4} \dot{\Delta}_{j}\left(\dot{S}_{k-1} u \dot{\Delta}_{k}\left(\partial_{i} P v\right)\right)+\sum_{|k-j| \leq 4} \dot{\Delta}_{j}\left(\dot{S}_{k-1}\left(\partial_{i} P v\right) \dot{\Delta}_{k} u\right)+\sum_{k \geq j-3} \dot{\Delta}_{j}\left(\dot{\Delta}_{k} u \tilde{\Delta}_{k}\left(\partial_{i} P v\right)\right) \\
& =I_{j}+I I_{j}+I I I_{j} .
\end{aligned}
$$

To prove this proposition, we can write

$$
\begin{aligned}
\left\|u \partial_{i} P v\right\| \|_{\mathcal{L}^{\rho}\left(I ; \dot{\mathcal{N}}_{p, \lambda, q}^{-2(\alpha-1)+\frac{3}{p^{\prime}}+\frac{\alpha}{\rho}+\frac{\lambda}{p}+\sigma}\right)} \lesssim & \left\{\sum_{j \in \mathbb{Z}} 2^{j\left(-2(\alpha-1)+\frac{3}{p^{\prime}}+\frac{\alpha}{\rho}+\frac{\lambda}{p}+\sigma\right) q}\left\|\widehat{I}_{j}\right\|_{L^{\rho}\left(I, \mathrm{M}_{p}^{\lambda}\right)}^{q}\right\}^{1 / q} \\
& +\left\{\sum_{j \in \mathbb{Z}} 2^{j\left(-2(\alpha-1)+\frac{3}{p^{\prime}}+\frac{\alpha}{\rho}+\frac{\lambda}{p}+\sigma\right) q}\left\|\widehat{I I}_{j}\right\|_{L^{\rho}\left(I, \mathrm{M}_{p}^{\lambda}\right)}^{q}\right\}^{1 / q} \\
& +\left\{\sum_{j \in \mathbb{Z}} 2^{j\left(-2(\alpha-1)+\frac{3}{p^{\prime}}+\frac{\alpha}{\rho}+\frac{\lambda}{p}+\sigma\right) q}\left\|\widehat{I I I}_{j}\right\|_{L^{\rho}\left(I, \mathrm{M}_{p}^{\lambda}\right)}^{q}\right\}^{1 / q} .
\end{aligned}
$$

We treat the above three terms differently. First, using Young's inequality (5) in Morrey spaces, and Lemma 6 with $|\gamma|=0$, we get

$$
\begin{aligned}
& \left\|\widehat{I}_{j}\right\|_{L^{\rho}\left(I, \mathrm{M}_{p}^{\lambda}\right)} \leq \sum_{|k-j| \leq 4}\left\|\dot{S}_{k-1} \widehat{u \dot{\Delta}_{k}\left(\partial_{i} P v\right)}\right\|_{L^{\rho}\left(I, \mathrm{M}_{p}^{\lambda}\right)} \\
& \leq \sum_{|k-j| \leq 4}\left\|\varphi_{k} \mathcal{F}\left(\partial_{i} P v\right)\right\|_{L^{\rho}\left(I, \mathrm{M}_{p}^{\lambda}\right)} \sum_{l \leq k-2}\left\|\varphi_{l} \hat{u}\right\|_{L^{\infty}\left(I, L^{1}\right)} \\
& \leq \sum_{|k-j| \leq 4}\left\|\varphi_{k} \mathcal{F}\left(\partial_{i} P v\right)\right\|_{L^{\rho}\left(I, \mathrm{M}_{p}^{\lambda}\right)} \sum_{l \leq k-2} 2^{l\left(\frac{3}{p^{\prime}}+\frac{\lambda}{p}\right)}\left\|\widehat{u}_{l}\right\|_{L^{\infty}\left(I, \mathrm{M}_{p}^{\lambda}\right)} \\
& \lesssim \sum_{|k-j| \leq 4} 2^{k \sigma}\left\|\widehat{v}_{k}\right\|_{L^{\rho}\left(I, \mathrm{M}_{p}^{\lambda}\right)}\left(\sum_{l \leq k-2} 2^{l(\alpha-1-\sigma) q^{\prime}}\right)^{\frac{1}{q^{\prime}}}\|u\| \mathcal{L}_{\mathcal{L}^{\infty}\left(I ; \mathcal{F} \dot{\mathcal{N}}_{p, \lambda, q}^{1-\alpha+\frac{3}{p^{\prime}}+\frac{\lambda}{p}+\sigma}\right)} \\
& \lesssim \sum_{|k-j| \leq 4} 2^{k(\alpha-1)}\left\|\widehat{v}_{k}\right\|_{L^{\rho}\left(I, \mathrm{M}_{p}^{\lambda}\right)}\|u\|{ }_{\mathcal{L}^{\infty}\left(I ; \mathcal{F} \dot{\mathcal{N}}_{p, \lambda, q}^{1-\alpha+\frac{3}{p^{\prime}}+\frac{\lambda}{p}+\sigma}\right)} .
\end{aligned}
$$

Multiplying by $2^{j\left(-2(\alpha-1)+\frac{3}{p^{\prime}}+\frac{\alpha}{\rho}+\frac{\lambda}{p}+\sigma\right)}$, and taking $l q-$ norm of both sides in the above estimate, we obtain $\left\{\sum_{j \in \mathbb{Z}} 2^{j\left(-2(\alpha-1)+\frac{3}{p^{\prime}}+\frac{\alpha}{\rho}+\frac{\lambda}{p}+\sigma\right) q}\left\|\widehat{I}_{j}\right\|_{L^{\rho}\left(I, \mathrm{M}_{p}^{\lambda}\right)}^{q}\right\}^{1 / q}$

$\lesssim\left\{\sum_{j \in \mathbb{Z}}\left(\sum_{|k-j| \leq 4} 2^{k\left(1-\alpha+\frac{3}{p^{\prime}}+\frac{\lambda}{p}+\frac{\alpha}{\rho}+\sigma\right)} 2^{(j-k)\left(-2(\alpha-1)+\frac{3}{p^{\prime}}+\frac{\alpha}{\rho}+\frac{\lambda}{p}+\sigma\right)}\left\|\widehat{v}_{k}\right\|_{L^{\rho}\left(I, \mathrm{M}_{p}^{\lambda}\right)}\right)^{q}\right\}^{1 / q} \times\|u\| \mathcal{L}^{\infty}\left(I ; \mathcal{F} \dot{\mathcal{N}}_{p, \lambda, q}^{1-\alpha+\frac{3}{p^{\prime}}+\frac{\lambda}{p}+\sigma}\right)$

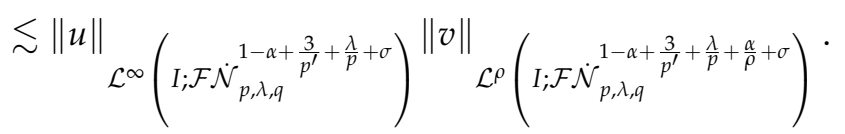

Likewise, we prove that

$$
\left\{\sum_{j \in \mathbb{Z}} 2^{j\left(-2(\alpha-1)+\frac{3}{p^{\prime}}+\frac{\alpha}{\rho}+\frac{\lambda}{p}+\sigma\right) q}\left\|\widehat{I I}_{j}\right\|_{L^{\rho}\left(I, \mathrm{M}_{p}^{\lambda}\right)}^{q}\right)^{1 / q} \lesssim\|v\|_{\mathcal{L}^{\infty}\left(I ; \mathcal{F} \dot{\mathcal{N}}_{p, \lambda, q}^{1-\alpha+\frac{3}{p^{\prime}}+\frac{\lambda}{p}+\sigma}\right)}\|u\| \mathcal{L}_{\mathcal{L}^{\rho}\left(I ; \mathcal{F} \dot{\mathcal{N}}_{p, \lambda, q}^{1-\alpha+\frac{3}{p^{\prime}}+\frac{\lambda}{p}+\frac{\alpha}{\rho}+\sigma}\right)}
$$



obtain

To evaluate $I I I_{j}$, we apply the Young inequality (5) in Morrey spaces and Lemma 6 with $|\gamma|=0$, we

$$
\begin{aligned}
& 2^{j\left(-2(\alpha-1)+\frac{3}{p^{\prime}}+\frac{\alpha}{\rho}+\frac{\lambda}{p}+\sigma\right)}\left\|\widehat{I I I}_{j}\right\|_{L^{\rho}\left(I, \mathrm{M}_{p}^{\lambda}\right)} \\
& \leq 2^{j\left(-2(\alpha-1)+\frac{3}{p^{\prime}}+\frac{\alpha}{\rho}+\frac{\lambda}{p}+\sigma\right)} \sum_{k \geq j-3} \sum_{|l-k| \leq 1}\left\|\mathcal{F}\left(\dot{\Delta}_{k} u \dot{\Delta}_{l}\left(\partial_{i} P v\right)\right)\right\|_{L^{\rho}\left(I, \mathrm{M}_{p}^{\lambda}\right)} \\
& \leq 2^{j\left(-2(\alpha-1)+\frac{3}{p^{\prime}}+\frac{\alpha}{\rho}+\frac{\lambda}{p}+\sigma\right)} \sum_{k \geq j-3|l-k| \leq 1}\left\|\widehat{u}_{k}\right\|_{L^{\rho}\left(I, \mathrm{M}_{p}^{\lambda}\right)}\left\|\varphi_{l} \mathcal{F}\left(\partial_{i} P v\right)\right\|_{L^{\infty}\left(I, L^{1}\right)} \\
& \leq 2^{j\left(-2(\alpha-1)+\frac{3}{p^{\prime}}+\frac{\alpha}{\rho}+\frac{\lambda}{p}+\sigma\right)} \sum_{k \geq j-3|l-k| \leq 1} 2^{l\left(\frac{3}{p^{\prime}}+\frac{\lambda}{p}\right)}\left\|\widehat{u}_{k}\right\|_{L^{\rho}\left(I, \mathrm{M}_{p}^{\lambda}\right)} 2^{l \sigma}\left\|\widehat{v}_{l}\right\|_{L^{\infty}\left(I, \mathrm{M}_{p}^{\lambda}\right)} \\
& \quad \sum_{k \geq j-3 l=-1} \sum^{1} 2^{\left(-2(\alpha-1)+\frac{3}{p^{\prime}}+\frac{\alpha}{\rho}+\frac{\lambda}{p}+\sigma\right)(j-k)} 2^{(\alpha-1) l}\left(2^{\left(-(\alpha-1)+\frac{3}{p^{\prime}}+\frac{\alpha}{\rho}+\frac{\lambda}{p}+\sigma\right) k}\left\|\widehat{u}_{k}\right\|_{L^{\rho}\left(I, \mathrm{M}_{p}^{\lambda}\right)}\right) \\
& \left.\quad \times \quad 2^{(l+k)\left(-(\alpha-1)+\frac{3}{p^{\prime}}+\frac{\lambda}{p}+\sigma\right)}\left\|\widehat{v}_{l+k}\right\|_{L^{\infty}\left(I, \mathrm{M}_{p}^{\lambda}\right)}\right) .
\end{aligned}
$$

Taking the $l^{q}$-norm on both sides in the above estimate and using Hölder's inequalities for series with $-2(\alpha-1)+\frac{\alpha}{\rho}+\frac{3}{p^{\prime}}+\frac{\lambda}{p}+\sigma>0$, we get

$$
\begin{aligned}
\left(\sum_{j \in \mathbb{Z}} 2^{j\left(-2(\alpha-1)+\frac{3}{p^{\prime}}+\frac{\alpha}{\rho}+\frac{\lambda}{p}+\sigma\right) q}\|\widehat{I I I}\|_{L^{\rho}\left(I, \mathrm{M}_{p}^{\lambda}\right)}^{q}\right)^{\frac{1}{q}} \\
\leq \quad\left(\sum _ { j \in \mathbb { Z } } \left(\sum_{m \leq 3} \sum_{l=-1}^{1} 2^{\left(-2(\alpha-1)+\frac{3}{p^{\prime}}+\frac{\alpha}{\rho}+\frac{\lambda}{p}+\sigma\right) m} 2^{(\alpha-1) l} 2^{\left(-(\alpha-1)+\frac{3}{p^{\prime}}+\frac{\alpha}{\rho}+\frac{\lambda}{p}+\sigma\right)(j-m)}\right.\right. \\
\left.\left.\quad \times\left\|\widehat{u}_{j-m}\right\|_{L^{\rho}\left(I, \mathrm{M}_{p}^{\lambda}\right)} 2^{\left(-(\alpha-1)+\frac{3}{p^{\prime}}+\frac{\lambda}{p}+\sigma\right)(j-m+l)}\left\|\widehat{v}_{j-m+l}\right\|_{L^{\infty}\left(I, \mathrm{M}_{p}^{\lambda}\right)}\right)^{q}\right)^{\frac{1}{q}} \\
\leq \sum_{l=-1}^{1} \sum_{m \leq 3} 2^{\left(-2(\alpha-1)+\frac{3}{p^{\prime}}+\frac{\alpha}{\rho}+\frac{\lambda}{p}+\sigma\right) m} 2^{(\alpha-1) l}\|u\|\left(I ; \mathcal{F} \dot{\mathcal{N}}_{p, \lambda, q}^{1-\alpha+\frac{3}{p^{\prime}}+\frac{\alpha}{\rho}+\frac{\lambda}{p}+\sigma}\right) \\
\quad \times\|v\| \\
\mathcal{L}^{\infty}\left(I ; \mathcal{F} \dot{\mathcal{N}}_{p, \lambda, \infty, \infty}^{1-\alpha+\frac{3}{p^{\prime}}+\frac{\lambda}{p}+\sigma}\right)
\end{aligned}
$$

Since $19 \hookrightarrow l^{\infty}$, we obtain

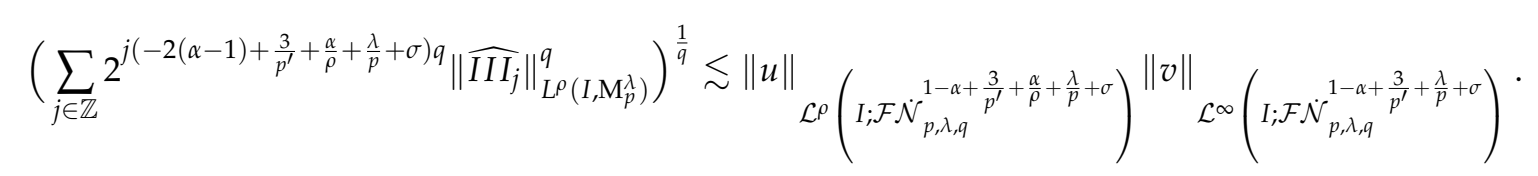

Estimates (10), (11), (12) and (13) yield (9) .

Lemma 9. Let $X$ be a Banach space with norm $\|\cdot\|_{X}$ and $B: X \times X \longmapsto X$ be a bounded bilinear operator satisfying

$$
\|B(u, v)\|_{X} \leq \eta\|u\|_{X}\|v\|_{X}
$$

for all $u, v \in X$ and a constant $\eta>0$. Then, if $0<\varepsilon<\frac{1}{4 \eta}$ and if $y \in X$ such that $\|y\|_{X} \leq \varepsilon$, the equation $x:=y+B(x, x)$ has a solution $\bar{x}$ in $X$ such that $\|\bar{x}\|_{X} \leq 2 \varepsilon$. This solution is the only one in the ball $\bar{B}(0,2 \varepsilon)$. Moreover, the solution depends continuously on $y$ in the sense: if $\left\|y^{\prime}\right\|_{X} \leq \varepsilon, x^{\prime}=y^{\prime}+B\left(x^{\prime}, x^{\prime}\right)$, and $\left\|x^{\prime}\right\|_{X} \leq 2 \varepsilon$, then

$$
\left\|\bar{x}-x^{\prime}\right\|_{X} \leq \frac{1}{1-4 \varepsilon \eta}\left\|y-y^{\prime}\right\|_{X}
$$

\section{Proof of theorem 4}

Proof. To ensure the existence of global solutions with small initial data, we will use Lemma 9. 
In the following, we consider the Banach space

$$
X=\mathcal{L}^{\infty}\left([0,+\infty) ; \mathcal{F} \dot{\mathcal{N}}_{p, \lambda, q}^{1-\alpha+\frac{3}{p^{\prime}}+\frac{\lambda}{p}+\sigma}\right) \cap \mathcal{L}^{1}\left([0,+\infty) ; \mathcal{F}_{p, \lambda, q}^{1+\frac{3}{p^{\prime}}+\frac{\lambda}{p}+\sigma}\right)
$$

First, we start with the integral equation

$$
\begin{aligned}
u & =e^{-\mu t \Lambda^{\alpha}} u_{0}+\int_{0}^{t} e^{-\mu(t-\tau) \Lambda^{\alpha}} \nabla \cdot(u(\tau) \nabla P u(\tau)) d \tau \\
& =e^{-\mu t \Lambda^{\alpha}} u_{0}+B(u, u) .
\end{aligned}
$$

We notice that $B(u, v)$ can be thought as the solution to the heat Equation (8) with $u_{0}=0$ and force $f=\nabla \cdot(u(\tau) \nabla P v(\tau))$. According to Lemma 7 with $s=1-\alpha+\frac{3}{p^{\prime}}+\frac{\lambda}{p}+\sigma$ and Proposition 8 with $\rho=1$, we obtain

$$
\begin{aligned}
& \|B(u, v)\|_{X} \leq\left(1+\left(\frac{4}{3}\right)^{\alpha}\right)\|\nabla \cdot(u \nabla P v)\| \underset{\mathcal{L}^{1}\left([0,+\infty) ; \mathcal{F} \dot{\mathcal{N}}_{p, \lambda, q}^{1-\alpha+\frac{3}{p^{\prime}}+\frac{\lambda}{p}+\sigma}\right)}{ } \\
& \leq\left(1+\left(\frac{4}{3}\right)^{\alpha}\right) C \mu^{-1}\|u\|_{X}\|v\|_{X} .
\end{aligned}
$$

By Lemma 9, we know that if $\left\|e^{-\mu t \Lambda^{\alpha}} u_{0}\right\|_{X}<R$ with $R=\frac{\mu}{4\left(1+\left(\frac{4}{3}\right)^{\alpha}\right) C}$

then the equation (14) has a unique solution in $B(0,2 R):=\left\{x \in X:\|x\|_{X} \leq 2 R\right\}$. To prove $\left\|e^{-\mu t \Lambda^{\alpha}} u_{0}\right\|_{X}<R$, notice that $e^{-\mu t \Lambda^{\alpha}} u_{0}$ is the solution to the dissipative equation with $u_{0}=u_{0}$ and $f=0$. So, Lemma 7 yields

$$
\left\|e^{-\mu t \Lambda^{\alpha}} u_{0}\right\|_{X} \leq\left(1+\left(\frac{4}{3}\right)^{\alpha}\right)\left\|u_{0}\right\|_{\mathcal{F N}_{p, \lambda, q}^{1-\alpha+\frac{3}{p^{\prime}}+\frac{\lambda}{p}+\sigma}} .
$$

If $\left\|u_{0}\right\| \underset{\mathcal{F} \dot{\mathcal{N}}_{p, \lambda, q}^{1-\alpha+\frac{3}{p^{\prime}}+\frac{\lambda}{p}+\sigma}}{ } \leq C_{0} \mu$ with $C_{0}=\frac{1}{4\left(1+\left(\frac{4}{3}\right)^{\alpha}\right)^{2} C^{\prime}}$, then (14) has a unique global solution $u \in X$ satisfying

$$
\|u\|_{X} \leq 2\left(1+\left(\frac{4}{3}\right)^{\alpha}\right)\left\|u_{0}\right\|_{\mathcal{F} \dot{\mathcal{N}}_{p, \lambda, q}^{1-\alpha+\frac{3}{p^{\prime}}}+\frac{\lambda}{p}+\sigma}
$$

\section{Proof of theorem 5}

Proof. To prove Theorem 5, we note $a(t, x):=e^{\mu \sqrt{t}|D|^{\frac{\alpha}{2}}} u(t, x)$. Using the integral Equation (14), we obtain

$$
\begin{aligned}
a(t, x)= & e^{\mu\left(\sqrt{t}|D|^{\frac{\alpha}{2}}-\frac{1}{2} t \Lambda^{\alpha}\right)} e^{-\frac{1}{2} \mu t \Lambda^{\alpha}} u_{0} \\
& +\int_{0}^{t} e^{\mu\left[(\sqrt{t}-\sqrt{\tau})|D|^{\frac{\alpha}{2}}-\frac{1}{2}(t-\tau) \Lambda^{\alpha}\right]} e^{-\frac{1}{2} \mu(t-\tau) \Lambda^{\alpha}} e^{\mu \sqrt{\tau}|D|^{\frac{\alpha}{2}}} \nabla \cdot(u \nabla(P u)) d \tau \\
:= & L u_{0}+\widetilde{B}(u, u) .
\end{aligned}
$$

In order to obtain the Gevrey class regularity of the solution, we use Lemma 9. Firstly, we start by estimating the term $L u_{0}=e^{-\frac{1}{2} \mu\left(\sqrt{t}|D|^{\frac{\alpha}{2}}-1\right)^{2}+\frac{\mu}{2}} e^{-\frac{1}{2} \mu t \Lambda^{\alpha}} u_{0}$.

Using the Fourier transform, multiplying by $\varphi_{j}$ and taking the $\mathrm{M}_{p}^{\lambda}$-norm we obtain

$$
\left\|\varphi_{j} \widehat{\operatorname{Lu}_{0}}\right\|_{\mathrm{M}_{p}^{\lambda}} \leq C e^{-\frac{1}{2} \mu t 2^{j \alpha}(3 / 4)^{\alpha}}\left\|\varphi_{j} \widehat{u_{0}}\right\|_{\mathrm{M}_{p}^{\lambda}} \text {. }
$$


Multiplying by $2^{j\left(1-\alpha+\frac{3}{p^{\prime}}+\frac{\lambda}{p}+\sigma\right)}$ and taking $l q$-norm we get

$$
\left\|L u_{0}\right\|_{\mathcal{L}^{\infty}\left([0,+\infty) ; \mathcal{F} \dot{\mathcal{N}}_{p, \lambda, q}^{1-\alpha+\frac{3}{p^{\prime}}+\frac{\lambda}{p}+\sigma}\right)} \leq C\left\|u_{0}\right\|_{\mathcal{F N}_{p, \lambda, q}^{1-\alpha+\frac{3}{p^{\prime}}+\frac{\lambda}{p}+\sigma}}
$$

Similarly

$$
2^{j\left(1+\frac{3}{p^{\prime}}+\frac{\lambda}{p}+\sigma\right)}\left\|\varphi_{j} \widehat{L u_{0}}\right\|_{L^{1}\left([0,+\infty) ; \mathrm{M}_{p}^{\lambda}\right)} \leq\left(\int_{0}^{\infty} e^{-\frac{1}{2} \mu t 2^{j \alpha}(3 / 4)^{\alpha}} 2^{j \alpha} d t\right) 2^{j\left(1-\alpha+\frac{3}{p^{\prime}}+\frac{\lambda}{p}+\sigma\right)}\left\|\varphi_{j} \widehat{u_{0}}\right\|_{\mathrm{M}_{p}^{\lambda}} .
$$

We conclude by taking $l^{q}$-norm that

$$
\mu\left\|L u_{0}\right\|_{\mathcal{L}^{1}\left([0,+\infty) ; \mathcal{F} \dot{\mathcal{N}}_{p, \lambda, q}^{1+\frac{3}{p^{\prime}}+\frac{\lambda}{p}+\sigma}\right)} \leq C\left\|u_{0}\right\|_{\mathcal{F N}_{p, \lambda, q}{ }^{1-\alpha+\frac{3}{p^{\prime}}+\frac{\lambda}{p}+\sigma}}
$$

Finally,

$$
\left\|L u_{0}\right\|_{X} \leq C\left\|u_{0}\right\| \underset{\mathcal{F} \dot{\mathcal{N}}_{p, \lambda, q}^{1-\alpha+\frac{3}{p^{\prime}}+\frac{\lambda}{p}+\sigma}}{ }
$$

On the other hand, we notice that $\widetilde{B}(u, v)$ as $\widetilde{B}\left(e^{-\mu \sqrt{\tau}|D|^{\frac{\alpha}{2}}} a, e^{-\mu \sqrt{\tau}|D|^{\frac{\alpha}{2}}} b\right)$ with $b:=e^{\mu \sqrt{\tau}|D|^{\frac{\alpha}{2}}} v$. Since $e^{\mu\left[(\sqrt{t}-\sqrt{\tau})|\xi|^{\frac{\alpha}{2}}-\frac{1}{2}(t-\tau)|\xi|^{\alpha}\right]}$ is uniformly bounded on $t \in(0, \infty)$ and $\tau \in[0, t]$, it sufficient to consider the estimate of $\left\|e^{\mu \sqrt{\tau}|D|^{\frac{\alpha}{2}}} u \partial_{i}(P v)\right\| \mathcal{L}^{1}\left(I ; \mathcal{F} \dot{\mathcal{N}}_{p, \lambda, q}^{2-\alpha+\frac{3}{p^{p}}+\frac{\lambda}{p}+\sigma}\right)$ for which we prove the flowing lemma.

Lemma 10. Let $1 \leq p<\infty, 1 \leq q \leq \infty, 0 \leq \lambda<3,1+\sigma<\alpha<\min \left\{2,2+\frac{3}{p^{\prime}}+\frac{\lambda}{p}+\sigma\right\}, I=[0, T), T \in(0, \infty]$, and set

$$
X=\mathcal{L}^{\infty}\left(I ; \mathcal{F} \dot{\mathcal{N}}_{p, \lambda, q}^{1-\alpha+\frac{3}{p^{\prime}}+\frac{\lambda}{p}+\sigma}\right) \cap \mathcal{L}^{1}\left(I ; \mathcal{F}_{\mathcal{N}_{p, \lambda, q}^{1+\frac{3}{p^{\prime}}+\frac{\lambda}{p}+\sigma}}\right)
$$

There exists a constant $C=C(p, q)>0$ depending on $p, q$ such that

$$
\left\|e^{\mu \sqrt{\tau}|D|^{\frac{\alpha}{2}}} u \partial_{i}(P v)\right\|_{\mathcal{L}^{1}\left(I ; \mathcal{F} \dot{\mathcal{N}}_{p, \lambda, q}^{2-\alpha+\frac{3}{p^{\prime}}+\frac{\lambda}{p}+\sigma}\right)} \leq C \mu^{-1}\|a\|_{X}\|b\|_{X}
$$

Proof. Based on the same procedure in the proof of Proposition 8, we evaluate the estimate of

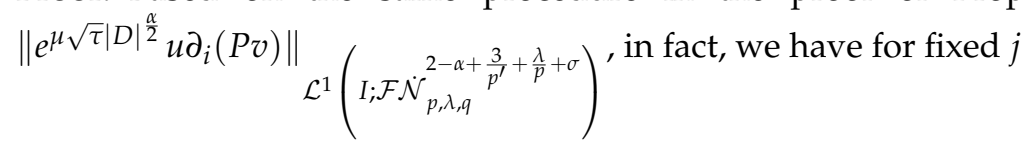

$$
\begin{aligned}
\dot{\Delta}_{j} e^{\mu \sqrt{\tau}|D|^{\frac{\alpha}{2}}}\left(u \partial_{i}(P v)\right)= & \sum_{|k-j| \leq 4} \dot{\Delta}_{j} e^{\mu \sqrt{\tau}|D|^{\frac{\alpha}{2}}}\left(\dot{S}_{k-1} u \dot{\Delta}_{k} \partial_{i}(P v)\right) \\
& +\sum_{|k-j| \leq 4} \dot{\Delta}_{j} e^{\mu \sqrt{\tau}|D|^{\frac{\alpha}{2}}}\left(\dot{S}_{k-1} \partial_{i}(P v) \dot{\Delta}_{k} u\right) \\
& +\sum_{k \geq j-3} \dot{\Delta}_{j} e^{\mu \sqrt{\tau}|D|^{\frac{\alpha}{2}}}\left(\dot{\Delta}_{k} u \widetilde{\Delta}_{k} \partial_{i}(P v)\right) \\
:= & S_{1, j}+S_{2, j}+S_{3, j} .
\end{aligned}
$$


Since $e^{\mu \sqrt{\tau}\left(|\xi|^{\frac{\alpha}{2}}-|\xi-\eta|^{\frac{\alpha}{2}}-|\eta|^{\frac{\alpha}{2}}\right)}$ is uniformly bounded on $\tau$ when $\alpha \in[0,2]$, we obtain

$$
\begin{aligned}
\left\|\widehat{S_{1, j}}\right\|_{\mathrm{M}_{p}^{\lambda}} & =\left\|\sum_{|k-j| \leq 4} \varphi_{j} e^{\mu \sqrt{\tau}|\xi|^{\frac{\alpha}{2}}} \mathscr{F}\left(\dot{S}_{k-1} u \dot{\Delta}_{k} \partial_{i}(P v)\right)\right\|_{\mathrm{M}_{p}^{\lambda}} \\
& =\left\|\sum_{|k-j| \leq 4} \varphi_{j} e^{\mu \sqrt{\tau}|\xi|^{\frac{\alpha}{2}}}\left[\left(\sum_{l \leq k-2} e^{-\mu \sqrt{\tau}|\xi|^{\frac{\alpha}{2}}} \widehat{a_{l}}\right) *\left(e^{-\mu \sqrt{\tau}|\xi|^{\frac{\alpha}{2}}} \mathscr{F}\left(\dot{\Delta}_{k} \partial_{i}(P b)\right)\right)\right]\right\|_{\mathrm{M}_{p}^{\lambda}} \\
& \left.=\| \sum_{|k-j| \leq 4} \varphi_{j} \int_{\mathbb{R}^{3}} e^{\mu \sqrt{\tau}\left(|\xi|^{\frac{\alpha}{2}}-|\xi-\eta|^{\frac{\alpha}{2}}-|\eta|^{\frac{\alpha}{2}}\right.}\right)\left(\sum_{l \leq k-2} \widehat{a_{l}}\right)(\xi-\eta) \mathscr{F}\left(\dot{\Delta}_{k} \partial_{i}(P b)\right)(\eta) d \eta \|_{\mathrm{M}_{p}^{\lambda}} \\
& \leq C\left\|\sum_{|k-j| \leq 4} \mathscr{F}\left(\dot{S}_{k-1} a \dot{\Delta}_{k} \partial_{i}(P b)\right)\right\|_{\mathrm{M}_{p}^{\lambda}} .
\end{aligned}
$$

The same calculus as in Proposition 8 gives

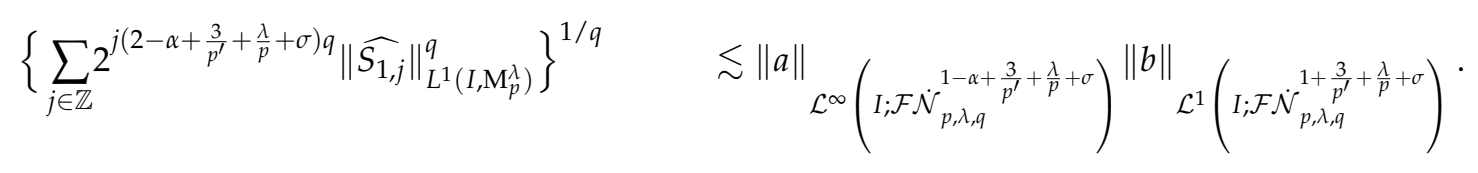

Similarly, we show that

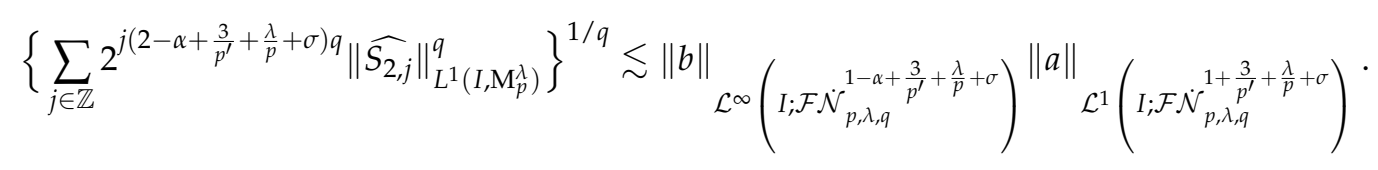

Similarly,

$$
\left\|\widehat{S_{3, j}}\right\|_{\mathrm{M}_{p}^{\lambda}} \leq \sum_{k \geq j-3} \sum_{|l-k| \leq 1}\left\|\mathcal{F}\left(\dot{\Delta}_{k} a \dot{\Delta}_{l}\left(\partial_{i}(P b)\right)\right)\right\|_{M_{p}^{\lambda}} .
$$

Using again the same procedure described in the proof of Proposition 8 we obtain

$$
\left\{\sum_{j \in \mathbb{Z}} 2^{j\left(2-\alpha+\frac{3}{p^{\prime}}+\frac{\lambda}{p}+\sigma\right) q}\left\|\widehat{S_{3, j}}\right\|_{L^{1}\left(I, \mathrm{M}_{p}^{\lambda}\right)}^{q}\right\}^{1 / q} \lesssim\|a\|{ }_{\mathcal{L}^{\infty}\left(I ; \mathcal{F} \dot{\mathcal{N}}_{p, \lambda, q}^{1-\alpha+\frac{3}{p^{\prime}}+\frac{\lambda}{p}+\sigma}\right)}\|b\| \mathcal{L}_{\mathcal{L}^{1}\left(I ; \mathcal{F} \dot{\mathcal{N}}_{p, \lambda, q}^{1+\frac{3}{p^{p}}+\frac{\lambda}{p}+\sigma}\right.} .
$$

Finally,

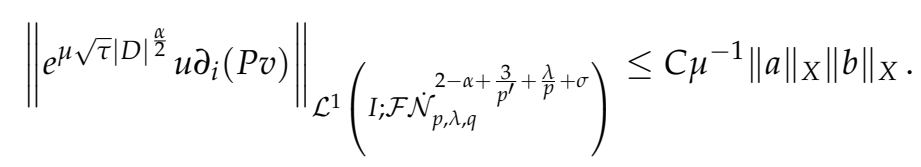

To finish the proof of Theorem 5 , it is easy to obtain the requested result by repeating the same step in the proof of Theorem 4 and Proposition 8.

Conflicts of Interest: "The author declare no conflict of interest."

\section{References}

[1] Zhou, X., Xiao, W., \& Zheng, T. (2015). Well-posedness and blowup criterion of generalized porous medium equation in Besov spaces. Electronic Journal of Differential Equations, 2015(261), 1-14.

[2] Caffarelli, L. A., \& Vázquez, J. L. (2011). Nonlinear porous medium flow with fractional potential pressure. Archive for Rational Mechanics and Analysis, 202 (2011), 537-565.

[3] Xiao, W., \& Zhou, X. (2016). On the generalized porous medium equation in Fourier-Besov spaces. arXiv preprint arXiv:1612.03304.

[4] Zhou, X., Xiao, W., \& Chen, J. (2014). Fractional porous medium and mean field equations in Besov spaces. Electron Journal fo Differential Equations, 2014(199), 1-14.

[5] Lin, F., \& Zhang, P. (2002). On the hydrodynamic limit of Ginzburg-Landau wave vortices. Communications on Pure and Applied Mathematics: A Journal Issued by the Courant Institute of Mathematical Sciences, 55(7), 831-856. 
[6] Biler, P., Imbert, C., \& Karch, G. (2011). Barenblatt profiles for a nonlocal porous medium equation. Comptes Rendus Mathematique, 349(11-12), 641-645.

[7] Blanchet, A., Carrillo, J. A., \& Masmoudi, N. (2008). Infinite time aggregation for the critical Patlak-Keller-Segel model in $R^{2}$. Communications on Pure and Applied Mathematics: A Journal Issued by the Courant Institute of Mathematical Sciences, 61(10), 1449-1481.

[8] Topaz, C. M., Bertozzi, A. L., \& Lewis, M. A. (2006). A nonlocal continuum model for biological aggregation. Bulletin of mathematical biology, 68(7), 1601-1623.

[9] Huang, Y., \& Bertozzi, A. L. (2010). Self-similar blowup solutions to an aggregation equation in $R^{n}$. SIAM Journal on Applied Mathematics, 70(7), 2582-2603.

[10] Li, D., \& Zhang, X. (2010). Global wellposedness and blowup of solutions to a nonlocal evolution problem with singular kernels. Communications on Pure E Applied Analysis, 9(6), 1591-1606.

[11] Bertozzi, A. L., \& Laurent, T. (2007). Finite-time Blow-up of Solutions of an Aggregation Equation in $R^{n}$. Communications in mathematical physics, 274(3), 717-735.

[12] Li, D., \& Rodrigo, J. L. (2010). Wellposedness and regularity of solutions of an aggregation equation. Revista Matemática Iberoamericana, 26(1), 261-294.

[13] Karch, G., \& Suzuki, K. (2010). Blow-up versus global existence of solutions to aggregation equations. Applied Mathematics (Warsaw), 38 (2011), 243-258.

[14] Laurent, T. (2007). Local and global existence for an aggregation equation. Communications in Partial Differential Equations, 32(12), 1941-1964.

[15] Chen, Q., \& Zhang, Z. (2007). Global well-posedness of the 2D critical dissipative quasi-geostrophic equation in the TriebelÜLizorkin spaces. Nonlinear Analysis: Theory, Methods E Applications, 67(6), 1715-1725.

[16] Wang, H., \& Zhang, Z. (2011). A frequency localized maximum principle applied to the 2D quasi-geostrophic equation. Communications in Mathematical Physics, 301(1), 105-129.

[17] Wang, W., \& Wu, G. (2018). Global mild solution of the generalized NavierÜStokes equations with the Coriolis force. Applied Mathematics Letters, 76, 181-186.

[18] Wu, G., \& Zhang, Q. (2013). Global well-posedness of the aggregation equation with supercritical dissipation in Besov spaces. ZAMM-Journal of Applied Mathematics and Mechanics/Zeitschrift für Angewandte Mathematik und Mechanik, 93(12), 882-894.

[19] Kato, T. (1992). Strong solutions of the Navier-Stokes equation in Morrey spaces. Boletim da Sociedade Brasileira de Matemática-Bulletin/Brazilian Mathematical Society, 22(2), 127-155.

[20] Cannone, M., \& Wu, G. (2012). Global well-posedness for NavierÜStokes equations in critical FourierÜHerz spaces. Nonlinear Analysis: Theory, Methods \& Applications, 75(9), 3754-3760.

[21] Ferreira, L. C., \& Lima, L. S. (2014). Self-similar solutions for active scalar equations in FourierÜBesovÜMorrey spaces. Monatshefte für Mathematik, 175(4), 491-509.

[22] El Baraka, A., \& Toumlilin, M. (2017). Global Well-Posedness for Fractional Navier-Stokes Equations in critical Fourier-Besov-Morrey Spaces. Moroccan Journal of Pure and Applied Analysis, 3(1), 1-13. 